\title{
Accessibility of Oral Healthcare Treatment Packages under Government Health Schemes in Rajasthan: A Critical Appraisal
}

\author{
Hansika Popli ${ }^{1}$, Manu Batra ${ }^{2}$, Deeksha Gijwani ${ }^{3}$, Drishti Chopra ${ }^{4}$, Nivedita Yadav ${ }^{5}$, Kartik Sokhal ${ }^{6}$
}

\begin{abstract}
Indian Government has introduced a variety of health insurance schemes with low or no premiums and significant sums insured in the hope of making good healthcare accessible to different strata of society. Rajasthan covers $10.4 \%$ of total nation's area with 7 th largest by the population. The state government has continuously strived toward providing health care to the population irrespective of the genre. The Rajasthan Government introduced Bhamasha Swasthya Bima Yojana (BSBY) in year 2015 which aimed at providing healthcare including major as well as minor dental procedures to the economic weaker section. Latest of all schemes is Ayushman Bharat Yojana. This article is about the accessibility of oral health care and treatment packages, their benefit to patients given under these two main schemes given by Government of Rajasthan. Keywords: Government, Health, Rajasthan.

Journal of Mahatma Gandhi University of Medical Sciences \& Technology (2021): 10.5005/jp-journals-10057-0184
\end{abstract}

\section{INTRODUCTION}

The Alma-Ata Declaration of 1978 emerged as a major milestone of the 20th century in the field of public health, and it identified primary health care as the key to the attainment of the goal "Health for All." In Western world the concept of providing health to all was initiated long back. In USA, Medicare and Medicaid, two government programs guaranteed health insurance, were formally enacted in 1965 as amendments (Titles XVIII and XIX, respectively) to the Social Security Act (1935) and went into effect in $1966 .{ }^{1}$

India being a developing country, have strived hard to achieve the ultimate health goal. Indian government has introduced a variety of health insurance schemes with low or no premiums and significant sums insured in the hope of making good healthcare accessible to different strata of society.

In India, there are various health insurance schemes at national and state level available like Employees State Insurance Scheme (ESIC,1952), Central Government Health Scheme (CGHS,1954), Aam Aadmi Bima Yojana (2007), Ayushman Bharat Scheme (2008), Rashtriya Swasthya Bima Yojana (2008), Chief Minister's Comprehensive Insurance Scheme (2012, Tamil Nadu), Karunya Health Scheme (2012, Kerala), Mahatma Jyotiba Phule Jan Arogya Yojana (2012, Maharashtra), Mukhyamantri Amrutam Yojana (2012, Gujarat), Yeshasvini Health Insurance Scheme (2013, Karnataka), Bhamashah Swasthya Bima Yojana (2015, Rajasthan), Pradhan Mantri Suraksha Bima Yojana (2015), Telangana State GovernmentEmployees and Journalists Health Scheme, West Bengal Health Scheme (2016 West Bengal), and Awaz Health Insurance Scheme (2017, Kerala). ${ }^{2}$

Rajasthan covers $10.4 \%$ of total nation's area with 7 th largest by the population. ${ }^{3}$ The state government has continuously strived towards providing health care to the population irrespective of the genre. Currently the following schemes in Rajasthan are operational- Mukhyamantri Nishulk Dava Yojna | Chief Minister's Free Medicine Scheme (2011), Mukhyamantri Nishulk Janch Yojna
${ }^{1-6}$ Department of Public Health Dentistry, Surendera Dental College and Research Institute, Sri Ganganagar, Rajasthan, India

Corresponding Author: Hansika Popli, Department of Public Health Dentistry, Surendera Dental College and Research Institute, Sri Ganganagar, Rajasthan, India, Phone: +91 9468566977, e-mail: drhansika02popli@gmail.com

How to cite this article: Popli H, Batra M, Gijwani D, et al. Accessibility of Oral Healthcare Treatment Packages under Government Health Schemes in Rajasthan: A Critical Appraisal. J Mahatma Gandhi Univ Med Sci Tech 2021;6(3):100-101.

Source of support: Nil

Conflict of interest: None

(2013), Nishulk Sanitary Napkins Distribution Scheme (2018) and Ayushman Bharat Mahatma Gandhi Rajasthan Swasthya Bima Yojana (AB-MGRSBY )(2020), and Mukhya Mantri Chiranjeevi Swasthya Bima Yojana (2021). ${ }^{4}$

The Rajasthan government introduced Bhamasha Swasthya Bima Yojana (BSBY) in year 2015 which aimed at providing healthcare including major as well as minor dental procedures to the economic weaker section sect.

The latest scheme; Ayushman Bharat Yojana, also known as the Pradhan Mantri Jan Arogya Yojana (PMJAY) was launched as recommended by the National Health Policy $2017,{ }^{5}$ on 23 September 2018, to achieve the vision of Universal Health Coverage (UHC), by the Central Government. ${ }^{6}$

Rajasthan government recently combined the Ayushman Bharat Pradhan Mantri Jan Arogya Yojana (AB-PMJAY) Bhamashah Swasthya Bima Yojana (BSBY), and launched a scheme Ayushman Bharat-Mahatma Gandhi Rajasthan Health Insurance Scheme (AB-MGRSBY).

On evaluation of treatment packages available under the two schemes, it was observed that though government is trying to

() The Author(s). 2021 Open Access This article is distributed under the terms of the Creative Commons Attribution 4.0 International License (https://creativecommons. org/licenses/by-nc/4.0/), which permits unrestricted use, distribution, and non-commercial reproduction in any medium, provided you give appropriate credit to the original author(s) and the source, provide a link to the Creative Commons license, and indicate if changes were made. The Creative Commons Public Domain Dedication waiver (http://creativecommons.org/publicdomain/zero/1.0/) applies to the data made available in this article, unless otherwise stated. 
enlarge the beneficiaries' base by bringing in more parameters of vulnerability for inclusion (even without a BPL card), under $A B-M G R S B Y$ scheme. Under the new scheme, although various major surgical procedures have been added but treatment packages related to dental care are missing. Only major oral and maxillofacial surgeries are available. Whereas in the BSBY scheme procedures like fracture of jaws, reduction and fixation were undertaken under BSBY as well along with other treatments like root canal treatment, curettage, complete denture, etc. Because of the omission of basic dental treatment in the new scheme has created a big void for the oral health care among the residents of Rajasthan.

Meanwhile the state government has started another health insurance scheme, that is, Mukhya Mantri Chiranjeevi Swasthya Bima Yojana (MMCSBY) which is started to strive towards Universal Health Insurance to all the residents of the state. In this scheme, individual will get health benefits by paying a nominal annual premium and for below poverty line population, it will be free. ${ }^{7-10}$ But the details of treatment under this scheme are still awaited as the scheme will be officially rolled out on $1^{\text {st }}$ May 2021.

\section{Conclusion}

Understanding the fervent public desire to have a genuinely useful healthcare system, especially for the have-nots of the society, the Government of Rajasthan has been reaching out to all with their flagship healthcare schemes. Our main concern is the neglect of basic dental treatments in AB-PMJAY which were there under the BSBY scheme.

The Central Government should ponder on including more extensive dental treatments in the AB-PMJAY scheme so that dental needs of the masses are met which if not done then can create a big gap which will not be easy to handle. The dental treatment should also be given utmost importance as other treatments.

\section{References}

1. Mazie, David M. "Medicare and Medicaid". Encyclopedia Britannica, 26-02-2020, https://www.britannica.com/topic/Medicare-UnitedStates-health-insurance. [Last accessed 13 April 2021.]

2. Types of government health insurance schemes in India. Aditya Birla Capital. 2020, https://www.adityabirlacapital.com/abc-of-money/ types-of-government-health-insurance-schemes-in-india [last accessed on 15-04-2021,10:34 am]

3. Rajasthan. Wikipedia.2021, https://en.wikipedia.org/wiki/Rajasthan. [last accessed on 15-04-2021, 10:37 am]

4. Government schemes in Rajasthan. Rajras. 2020, https://www. rajras.in/rajasthan/government/schemes [last accessed 15-042021,10:26 am]

5. About Pradhan Mantri Jan Arogya Yojana(PM-JAY). National Health Authority. 2018, https://pmjay.gov.in/about/pmjay [last accessed on 15-04-2021, 10:41 am]

6. PMJAY-All about Ayushman Bharat Yojana, Eligibility \& Application Process. Bajaj Finserv. 2020, https://www.bajajfinserv.in/insights/ ayushman-bharat-yojana-are-you-eligible-for-the-pmjay-scheme\# [last accessed on 15-04-2021, 10:44 am]

7. Ayushman Bharat Mahatma Gandhi Rajasthan Swasthya Bima Yojana. Rajras. 2021, https://www.rajras.in/ayushman-bharat-mahatmagandhi-rajasthan-swasthya-bima-yojana-ab-mgrsby/ [last accessed on 13-04-2021, 11:41 am]

8. Bhamashah Swasthya Bima Yojana by Government of India. Bank Bazaar. 2021, https://www.bankbazaar.com/healthinsurance/bhamashah-swasthya-bima-yojana.html [last accessed on 15-04-2021, 10:53 am]

9. Rajasthan Mukhyamantri Chiranjeevi Yojana will start from April 1, registration will be like this. Patrika. 31 March 2021, https://www.patrika.com/jaipur-news/rajasthan-mukhyamantrichiranjeevi-yojana-start-from-1-april-6773459/ [last accessed on 15-04-2021, 11:14]

10. Mukhyamantri Chiranjeevi Yojana Rajasthan 2021 CM 5 lakh health insurance scheme registration. Shiksha Crida. 2021, https://crida. in/mukhyamantri-chiranjeevi-yojana-rajasthan/ [last accessed on 15-04-2021, 11:20 am] 